Jean-Paul Narcy-Combes

Université Sorbonne Nouvelle - Paris 3, France https://orcid.org/0000-0001-8318-8501 jean-paul.narcy-combes@wanadoo.fr

\title{
COMMENT LA RÉFLEXION SUR LE TRANSCULTURING CONDUIT À REPENSER LA COMPÉTENCE INTERCULTURELLE
}

\author{
How reflecting on transculturing leads \\ to reconsidering intercultural competence
}

This article deals with the link between transculturing and translanguaging and what this link reveals. The ensuing transdisciplinary analysis shows that so-called intercultural competence results from the same emotional and cognitive processes as the theory of mind (social comprehension) that we all develop to relate to others. Turning to social psychology may then lead to more effective pedagogical approaches to "intercultural competence".

Keywords: transculturing, translanguaging, emotions, cognition, intercultural competence

Słowa kluczowe: transkulturowość, transjęzyczność, emocje, myślenie, kompetencja międzykulturowa

\section{Introduction}

Le parallélisme entre ce que certains appellent transculturing (comportements transculturels) et le construit de translanguaging (production translangagière) a éveillé notre intérêt. Pour García (2009), la production translangagière est un ensemble de processus qui englobe de multiples pratiques discursives et qui est la norme dans les communautés multilingues. Wei (2017) 
le décrit comme l'ensemble des performances linguistiques des locuteurs plurilingues, leur permettant de dépasser leurs limites dans les codes qu'ils utilisent par un jeu d'alternance entre eux dans la combinaison des structures et des formes pour exprimer leurs messages, leurs valeurs et leurs identités et préciser leurs relations. Néanmoins, le discours peut être quelquefois monocode, ce qui ne signifie pas qu'il y a unicité culturelle en amont, comme Wei (2017) nous le montre avec un corpus de New Chinglish, fréquent à Singapour. Les énoncés ou termes sont en anglais courant, ou cohérent avec le lexique de l'anglais, avec une connotation spécifique pour des locuteurs du chinois, ou des locuteurs de l'anglais en contact avec des chinois. On y trouve :

- Smilence: smile plus silence (réaction stéréotypée des chinois).

- Chinsumer: mélange de 'Chinese consumer' (acheteur compulsif de produit de luxe).

Du côté francophone, de tels phénomènes sont repérés au Liban également, par exemple, (Wehbe, 2017).

Cette contribution part d'une recherche initiée dans Narcy-Combes JeanPaul (2018). Une réflexion théorique sur la cognition et la personnalité (NarcyCombes et Narcy-Combes, 2019) nous a sensibilisés à nouveau au rôle de la théorie de l'esprit (voir infra) que notre domaine mentionne peu. La soutenance de Judith White (2018) a lancé un débat qui nous a conduit à affiner notre compréhension de la façon dont les individus gèrent leurs rencontres avec autrui. Nous voudrions aller au-delà du langage : nous postulons que toute production translangagière révèle des processus internes antérieurs au discours qui en est une conséquence, et ce sont les circonstances qui déterminent le choix du ou des codes (Grosjean, 2008). Nous nous plaçons au niveau du vécu où le discours véhicule des expériences multiples qui l'expliquent et nous avançons que la façon dont les individus gèrent leur discours et leurs comportements révèle leur complexité individuelle et, de ce fait, culturelle. Pour nous, les comportements transculturels (Baena, 2006) expliquent les productions translangagières et cet article poursuivra la réflexion entamée dans Narcy-Combes et Narcy-Combes (2019). Nous partirons de l'énaction, pour nous tourner ensuite vers l'action et son lien avec l'identité et la façon dont celle-ci se construit dans nos interactions dans des contextes multiples. Nous verrons si les interactions langagières sont décrites comme le reflet d'une pluralité culturelle et langagière.

Nous nous appuierons sur plusieurs auteurs, dont Dervin (2011) et Baena (2006), à qui nous avons emprunté le terme transculturing ${ }^{1}$. D'autres références soulignent la complexité de ces phénomènes. Saïd (2002) décrit la

\footnotetext{
${ }^{1}$ Nous parlons de comportements transculturels. Notre définition de transculturel de ce fait ne correspond pas à celle que donne Puren dans son article dans ce numéro.
} 
souffrance qu'ils peuvent causer et des études s'arrêtent sur la vulnérabilité qu'ils créent (Moro, 2010). Citons L'autre ${ }^{2}$, une revue qui explore la clinique transculturelle dans les interactions entre psychisme, cultures et sociétés ce qui conduit à interroger le statut de la langue, de la culture, des théories, des dispositifs de soins voire des politiques de santé. Sans aller aussi loin, nous parcourrons de nombreux champs théoriques dans le but de comprendre les expériences transculturelles pour aboutir à une position nouvelle sur la compétence dite interculturelle.

\section{Réflexion transdisciplinaire}

L'adjectif se justifie car l'étude se trouve au croisement de plusieurs disciplines, la sociologie, l'anthropologie, la psychologie, les sciences de la cognition, et bien sûr, les sciences du langage (voir Narcy-Combes M.-F., 2018) pour résoudre les problèmes que pose l'intervention sur le terrain de l'enseignement (Narcy-Combes et Narcy-Combes, 2019). Une revue des apports de ces domaines en ce qui concerne les comportements transculturels et aux conséquences que ces croisements transdisciplinaires nous ont permis de clarifier notre compréhension de ces phénomènes.

\subsection{L'énaction}

Notre position en ce qui concerne la cognition se concentrera ici synthétiquement sur ce que Varela (1993) postule au niveau de la corporéité de l'intelligence cognitive. Dans le modèle qu'il propose (l'énaction), une cognition, n'est pas la représentation d'un monde pré-donné, mais la construction conjointe d'un monde et d'un esprit à partir de ce que vit l'individu. Cependant ce monde lui reste spécifique, préciserons-nous, et ne correspond pas à ce que d'autres ont construit, ce qui interpelle le construit de culture partagée.

\subsection{L'action et les différents types d'action}

Enaction inclut le mot action, selon Goffman (1974) c'est par elle que nous réalisons et déterminons notre identité. Les actions (une action est rarement isolée), impliquent un travail de planification, de distribution et de coordination, et suscitent des émotions, des problèmes, des conflits qui conduisent à en modifier le cours et à nous transformer. Toute action éveille chez l'individu une forme de conscience de ses limites, de ses capacités, et de sa relation avec l'objet et avec

2 https://www.transculturel.eu/Presentation-de-la-revue-L-autre-Cliniques-cultureset-societes-Publications-transculturelles_a203.html , accès le 24/12/ 2018. 
les éléments du contexte qui entrent en jeu (Rabardel, 2005). On peut distinguer (voir Goffman, 1974 ; Leontiev, 2009 ; Narcy-Combes, 2005, par ex.) :

1. I'action consciente, mobilisant des outils et des connaissances face à une nouvelle situation ;

2. l'opération, automatisée, mais attendue dans le cadre d'une grande action complexe programmée ;

3. la réaction, lors d'un événement, moins programmé. Elle est plus ou moins consciente et « imprimée » en raison de rencontres préalables de situations similaires et répond à une visée moins précise, voire non-consciente (Narcy-Combes, 2005).

Ceci nous conduit à réfléchir également en termes de visées, de désirs, de valeurs, d'objectifs, et sans doute, de ce fait, d'intention.

\subsection{Intention et intentionnalité}

Le dessein (plus ou moins délibéré), le niveau de l'intention permet de différencier action, opération et réaction. L'intention résulte initialement de l'interaction entre l'individu et l'environnement dans le but d'agir (Xue, 2016). Elle a des racines profondes dans la personnalité de cet individu (visées, désirs, valeurs) (Clot, 2008). Elle peut être collective. Chacun des intervenants a un parcours personnel unique qui a construit sa subjectivité (LeDoux, 2003). Il y a autant de variations individuelles que d'intervenants malgré des points communs en raison de la proximité des environnements que le groupe partage (LeDoux, 2003). Dans l'opération ou la réaction, ce serait plutôt une intentionnalité qui gouverne : une façon moins affirmée, moins consciente, de viser un résultat (Scheler, 2003).

Au-delà de l'intentionnalité et des intentions que chacun a définies, des situations imprévues se produisent, qui déclenchent des (ré)actions différentes de ce qui a été planifié, en fonction, ou non, des convictions et des objectifs qui ont été annoncés. Dans l'urgence ou dans la surprise, l'individu ne pense pas de la façon dont il verbalise sa pensée (Cicurel et Narcy-Combes, 2014). Par l'analyse de verbalisations suite à la réaction (auto-confrontation), Xue (2016) montre que ces réactions sont conditionnées par soit :

1. la personnalité même de l'individu (à son insu),

2. des émotions issues des contraintes que l'institution ou le contexte déclenchent à ce moment-là,

3. son intentionnalité profonde.

Quand les conditions ne correspondent pas aux attentes, des problèmes surgissent qui nécessitent une remobilisation des connaissances et des outils, et les (ré)actions dépendent alors du jeu réciproque de l'intensité des émotions, du recul, de l'expérience de chacun et de son intentionnalité profonde, non sans 
conflits internes. Ce qu'il a vécu dans l'enfance conditionne largement la façon dont il réagit lors de problèmes socio-émotionnels (Okon-Singer et al., 2015). Les humains attribuent souvent l'intensité de l'émotion ou de la motivation aux événements externes alors que leur pouvoir motivationnel provient en partie de la décharge en dopamine qui survient à ce moment et qui est une réaction physiologique conditionnée (Schultz et al., 2000). Dans un environnement peu familier, des émotions inopportunes peuvent être déclenchées et conduire à des ressentis ou des comportements inadéquats dont il serait utile de devenir conscient. Jardou (2018) souligne que lors d'expériences de mobilité, c'est surtout lors des rencontres informelles de la vie quotidienne que l'incompréhension est la plus grande et les émotions le plus intenses. On mesure l'importance du recul qui permet à la fois de mieux se comprendre et de mieux comprendre les autres (Channouf, 2004), il convient donc de comprendre nos fonctionnements inconscients.

\subsection{L'inconscient cognitif}

L'individu n'agit donc pas toujours en pleine conscience, ce qui, d'une certaine manière, lui facilite la vie. Norman (1993) décrit deux types de modes cognitifs :

1. le mode expérimental où les (ré)actions se produisent de façon efficace et sans effort (les opérations) et où la personne ne se pose pas de problème;

2. le mode réflexif, où elle mobilise ses connaissances et ses expériences pour trouver de nouvelles solutions, ce qui implique la présence de raisonnement, de prise de décision et de créativité.

Nous ajouterions que cette prise de conscience pour modifier les comportements n'est possible que s'il n'y a ni urgence, ni pression.

Certaines (ré)actions résultent de l'urgence, de ce fait, le degré de réflexivité actionnelle diminue (Perrenoud, 1996), voire s'éteint (on n'est plus dans le cognitif). Néanmoins, les réactions révèlent des visées qui ne sont pas toujours claires et se manifestent dans la direction et l'intensité de ce que chacun fait (Channouf, 2004). Certains parlent d'un inconscient cognitif qui conduit les opérations (et les réactions), même dans des situations cognitivement complexes telles que la pratique professionnelle (incorporation de l'action, voir Lenoir, 2007 ; Varela, 1993). La question se pose de savoir comment cet inconscient cognitif se met en place, ce qui nous mène à explorer la façon dont chacun se construit dans les environnements sociaux où il est amené à interagir.

\subsection{Un développement social et situé}

Pour le socioconstructivisme, le développement cognitif de l'individu se fait dans l'interaction avec les autres participants avec lesquels se met en place 
un partage de connaissances et de cultures (Vygotski, 1993). Les théories neurobiologiques et psychologiques confirment ce rôle du contexte (et donc de ce que l'on appelle la/les culture/s) dans l'émergence de l'action et de la cognition humaines. Des signes parfois imperceptibles réveillent des sensations vécues dans des situations similaires qui suscitent des prédictions (Dehaene, 2011) qui vont déclencher une/des (ré)action/s proche/s de celles déclenchées dans ces situations initiales (Freze et Zapf, 1994). Toute différence ultérieure amène à réagir et à résoudre les problèmes en fonction du degré d'urgence et des émotions ressenties, là aussi plus ou moins consciemment. Trois questions se posent auxquelles il est important de pouvoir répondre :

1. Quelle est l'origine de ces signes ?

2. Quelle est la persistance de cette similitude ?

3. Comme il y a nécessairement plusieurs choix, que se passe-t-il ? Répondre à ces questions implique de comprendre les rapports entre ce qui relève de l'inconscient et de la réflexivité.

\subsection{Automaticité comportementale et réflexivité}

Le cerveau est, apparemment, en pilotage automatique tant que rien ne le perturbe. C'est le niveau de perturbation (urgence, surprise, etc.) mais aussi la réflexivité telle que la définit Norman (1993) qui activent la conscience. Ce peut être :

1. une tâche nouvelle,

2. le besoin de résoudre un problème nouveau,

3. une insatisfaction par rapport à ce que l'on vit, sans urgence,

4. une urgence (si nous ne sommes pas trop perturbés).

L'activité neuronale est alors relativement intense. Elle baisse avec la maîtrise de la tâche, puis s'affaiblit avec l'automatisation du contrôle des opérations accompagnée d'un taux de vigilance ou de réflexivité soumis à la façon dont l'individu évalue la situation (Freze et Zapf, 1994). Un lien est établi entre les affects et la conscience.

Ces fonctionnements correspondent également aux théories émergentistes (dont l'énaction) pour lesquelles l'humain se construit dans une interaction avec l'environnement et suit ainsi l'évolution de celui-ci pour mobiliser d'autres organismes physiologiques afin de s'adapter et de réagir (Kaptelinin, 1996). Chacun peut évoluer et ré-automatiser ses comportements ou faire des choix en fonction de la façon dont il vit les interactions. Il reste à comprendre comment, en particulier lors des changements d'environnements. C'est le langage qui sépare la simple expérience de la capacité de réfléchir sur elle. Langage et réflexivité seraient apparus conjointement chez l'homme primaire. Dans la vie quotidienne, la plus grande partie de l'expérience reste primaire, pas 
réflexive (Varela, 1993). Dans des environnements inhabituels cela peut créer des problèmes. On peut se demander si cela signifie que nous ne pensons pas, même dans de telles situations, sans en avoir conscience. Nous avons donc besoin de comprendre comment fonctionne la pensée.

\subsection{Les liens entre la conscience, la pensée et le langage}

La conscience est issue du système émotionnel pour résoudre le besoin d'un lien entre les informations sensorielles, attachées à un évènement donné alors que ces informations dépendent de réseaux différents, intervenant dans des zones différentes du cerveau, sans liaison neuronale directe entre eux (Damasio, 2010). Selon les théories actuelles, le cerveau ne stocke pas les données, mais crée des réseaux qui, lorsqu'ils sont activés, permettent de relancer les réactions appropriées (Rousset, 2000). Un souvenir n'est donc qu'une reconstruction. Différentes zones du cerveau qui ont reçu l'information l'ont encodée au même moment, la reconstruisent en synergie à partir d'un indice déclencheur. Selon Damasio (2010), les neurobiologistes s'accordent sur le fait que la conscience a une fonction adaptative et apparaît lors de comportements complexes quand les automatismes ne sont pas en place, sont inopérants et qu'un besoin d'adaptation est ressenti.

Nous avons vu précédemment que le conditionnement/l'automaticité, le passage au réflexif, est modifiable, non sans un nouvel entraînement qui va au-delà du savoir. Le réflexif est lourd cognitivement et émotionnellement (Xue, 2016). Les émotions ont une fonction cognitive que les humains contrecarrent fréquemment par un jeu de stratégies implicites ou explicites que l'on appelle mécanismes de défense (Payen, 2011): évitement, occultation, projection, etc. Ces derniers ne sont pas intentionnels mais semblent fonctionner sans effort et sans planification, malgré un coût émotionnel ou cognitif important. Les stratégies explicites exigent un degré de contrôle plus évident et dépendent de la situation émotionnelle (idem et Xue, 2016).

Par conséquent, le plus grand obstacle au changement vient du fait que le comportement conditionné nous protège à notre insu de toute déstabilisation, pas nécessairement de façon adéquate. La sélectivité socio-émotionnelle (Guillaume et al., 2009) et l'absence de motivation conduisent à se protéger dans l'évitement, le rejet ou l'occultation. Nos préférences émotionnelles sont conditionnées par l'impression relative et subjective du temps que l'on a devant nous, mais aussi par les bénéfices que l'on peut attendre de notre action. Quand les perspectives de temps sont limitées et/ou les bénéfices peu évidents, les individus peuvent privilégier les actions qui déclencheront des émotions agréables avec des gratifications immédiates (ibidem : 249). 
La neuro-imagerie ne permet pas d'observer la pensée dans le cerveau, elle est inférée et reconstruite à partir des comportements et dans les réalisations comportementales, artistiques, scientifiques qui sont observables. Dans le cerveau, on n'observe que des jeux de réactions biologiques (activité électrique, variation du flux sanguin) qui correspondent à ce qui se passe quand on dit qu'un individu pense. Le cerveau s'appuie sur les ressemblances plus que sur les différences. La mise en correspondance des traits communs entre les phénomènes qui intervient à chaque étape lorsque nous interprétons, décidons, agissons, nous remémorons, etc., génère l'analogie. Celle-ci fonctionne dans toutes les sphères de la cognition (perception, mémoire, langage, apprentissage), pour constamment établir des liens entre les concepts et l'environnement, entre le passé et le présent de chaque individu et établir des catégories qui à leur tour servent à organiser le réel (avec des risques de projection). Contrairement à ce que beaucoup pensent, la pensée véritable procède par bifurcation et par sérendipité que l'on peut comprendre comme la capacité de découvrir par hasard : on fait une analogie avec quelque chose qui n'était pas prévu et donc on trouve ce qu'on ne cherchait pas (voir, par ex. Serres, 2015). La pensée est processuelle, elle ne devient cognitive que lorsque le discours intérieur ou extérieur en a donné forme et conscience et on ne peut comprendre, décrire et expliquer sa pensée sans se référer à son contexte individuel, culturel et social. Vygotski (1934) ajoute : la pensée n'est pas seulement exprimée par les mots, elle vient à l'existence à travers les mots, nous dirions qu'elle devient consciente. La conscience, de ce fait, est le résultat de la « rencontre » de la pensée et du langage. Pour Bakhtine (1977), le langage et la conscience ne peuvent être différenciés.

Il est personnellement et socialement utile de devenir conscient du fait que nos pensées et nos constructions sont personnelles et que les autres ne réagissent pas et ne construisent pas le monde comme nous. Un domaine de réflexion s'y est intéressé que nous allons aborder.

\subsection{La théorie de l'esprit}

La cognition sociale (Duval et al., 2011) nous dit que chacun acquiert une théorie de l'esprit de lui-même et des autres qui lui donne l'aptitude cognitive d'interpréter (plus ou moins adéquatement) les comportements en termes d'états mentaux à partir de ses représentations de la vie personnelle et sociale (Callaghan et al., 2005). On parle aussi de "compréhension sociale", et souvent d' " empathie ». Selon Berthoz (2015 : 22) celle-ci suppose, qu'on soit capable d'éprouver l'émotion telle qu'elle est ressentie par autrui en se mettant effectivement à sa place. Les humains s'appuient plus ou moins explicitement 
sur ces sentiments afin de savoir comment se comporter dans les situations sociales qu'ils vivent.

Les enfants se construisent implicitement une théorie de l'esprit qui leur permet de voir que les personnes agissent en fonction de leurs croyances et de leurs sentiments qui peuvent être différents des leurs. Plusieurs recherches confirment le rôle de cette construction dans le développement cognitif, langagier et social et soulignent qu'elle est d'ordre culturel en fonction des rencontres et des expériences rencontrées (par ex. Bender \& Beller, 2013 ; Shahaeian et al., 2014).

La théorie de l'esprit conduit à deux types de représentations :

1. Celles de premier ordre sont celles que l'on construit de l'état mental d'une personne en adoptant sa perspective. Elles permettent de comprendre que l'autre a des représentations plus ou moins pertinentes et que chacun a une conscience propre et spécifique.

2. Celles de deuxième ordre sont celles qu'une personne construit sur les représentations d'une autre personne. Ce niveau exige des ressources cognitives plus importantes pour arriver à une compréhension plus fine du comportement humain.

L'attribution d'états mentaux résulte de deux mécanismes fonctionnels qui permettent de déterminer l'état mental d'une personne :

1. Le décodage des états mentaux, affectifs ou cognitifs est lié à la perception et l'identification d'informations sociales et d'indices présents dans l'environnement (la prosodie, par exemple). Ce mécanisme de décodage serait automatique, spontané et pré-conceptuel et dépendrait donc de la perception, impliquant surtout des processus primaires : percevoir un sarcasme résulte de la détection simultanée d'indices prosodiques, émotionnels et comportementaux.

2. Le processus de raisonnement permet de comprendre, d'expliquer ou de prédire les actions et impose d'avoir accès aux connaissances ou aux faits concernant soit le protagoniste, soit les circonstances contextuelles. Ceci implique des fonctions de plus haut niveau que le décodage et permet de séparer plaisanterie et mensonge, de deviner comment l'autre va réagir, etc. Des processus cognitifs complexes sont déclenchés en parallèle avec d'autres fonctions cognitives, d'où la difficulté d'analyser séparément les états mentaux (on est proche du métaculturel que décrit Puren dans ce numéro).

Ces mécanismes, comme tout mécanisme, sont largement conditionnés et déclenchent des réactions qui dépendent du vécu antérieur et ne sont pas nécessairement adaptées à des situations inconnues.

Dehaene (2013, p. 323) postule que l'introspection et la théorie de l'esprit sont deux facettes d'un même système de représentation mentale qui 
joue un rôle essentiel dans le dialogue social propre à l'espèce humaine. Le langage et la théorie de l'esprit entretiennent une relation d'interactions bidirectionnelles en lien avec la mémoire épisodique. Une relation est également avancée entre mémoire autobiographique et capacités d'inférence. II est clair que l'apprentissage de langues additionnelles et/ou la vie dans un environnement nouveau seront facilités par une théorie de l'esprit efficace et donc adaptée. Émerge ainsi un lien avec ce que l'on appelle la compétence interculturelle (Chang, 2017) que nous comprendrons mieux si nous nous tournons d'abord vers le rôle du discours dans l'activité sociale.

\subsection{Agir avec le langage}

Le discours est l'instrument clé de la réalisation de l'activité sociale, il permet de dépasser les problèmes qui viennent du fait que chacun a des visions différentes de la situation. Comme toute action, l'agir communicationnel est contextuellement, socio-culturellement, historiquement et personnellement marqué (Habermas, 1984).

Pour communiquer, nous avons besoin, dans un premier temps, d'établir un code commun (Knight \& Power, 2011). Tout discours reflète les spécificités de la communauté socioculturelle ou professionnelle qui le produit (Halliday, 1975). L'hypothèse Sapir-Whorf (Sapir, 1985) soulignait déjà le lien entre environnement, cognition et discours : la langue reflète l'environnement dans lequel elle prend forme et qui la délimite (Sapir, 1985). Cette hypothèse relativiste s'est maintenue sous une forme dite faible (Gumperz et Levinson, 1996). Le discours, produit social, est donc caractérisé et limité par le milieu où il s'est construit (Wittgenstein, 1980). Quels sont les effets des contacts dans des contextes différents pour un même individu ? Nous abordons ainsi les phénomènes dits « culturels ».

\subsection{Quand le culturel rencontre le personnel, le construit de transculturing : nos appuis}

Ce qui précède nous permet de comprendre pourquoi les chercheurs en ethnologie contestent la notion de culture monolithique (Descola, 2005). Baena (2006) s'arrête sur les effets de vécus pluriculturels sur les comportements et les pensées, et propose une analyse transculturelle pour comprendre les dimensions dynamiques de la conscience. En employant "transculturing" pour parler de la façon de vivre des expériences complexes ou apparaissent des choix non déterminés, Baena souligne l'importance de saisir le dynamisme de l'expérience pluriculturelle sans la stabiliser en éléments permanents et inclut tout ce 
qui a trait à la vie humaine. De ce fait, des chercheurs (Puren, 2002 ou ce numéro, ou Dervin, 2011) se focalisent davantage sur la co-construction culturelle dans les interactions qui relève de phénomènes actifs que sur l'interculturel.

Dervin (2011) met en évidence l'absence des stéréotypes analytiques des identités et des cultures dans les discours de couples mixtes où il apparait que les comportements, ainsi que les propos sur les langues, les identités et les cultures sont des constructions instables liées à l'instant, traduisant la pluralité des individus. Notre réflexion sur l'énaction permet de comprendre que ces constructions ne peuvent qu'évoluer selon le vécu des individus, en particulier si les environnements qu'ils connaissent sont différents les uns des autres.

\subsection{Pluralité des habitus}

Lahire (2001) nous a sensibilisés à la pluralité de l'habitus. Si le cerveau humain fonctionne par conflits entre le système rationnel (plus récent) et le système émotionnel (très ancien) (Eagleman, 2012) pour activer un comportement adapté à une situation, surtout si elle est inhabituelle ou si l'enjeu est grand (voir plus haut), ces conflits sont complexifiés lorsqu'un individu a des références culturelles plurielles, et cela explique le travail des psychologues et des psychiatres que nous avons mentionné plus haut. Nous serions tenté de dire que les individus vivent des expériences pluriculturelles mais réagissent transculturellement, selon notre définition de ce construit, c'est-à-dire que leurs comportements sont en quelques sortes métissés en fonction de leurs émotions et de leurs interprétations des situations. Les émotions ont aussi leur rationalité qui est parfois supérieure aux arguments logiques (Damasio, 2010). Il existe une autre source de conflits internes : la mémoire. Certes, ce qu'il a vécu dans l'enfance conditionne la façon dont un individu réagit lors de problèmes socio-émotionnels, mais les souvenirs ordinaires de la vie quotidienne sont stockés dans les neurones par I'hippocampe, tandis que les souvenirs traumatisants le sont dans l'amygdale et sont difficiles à effacer et susceptibles de ressurgir dans certaines situations difficiles à gérer. On comprend l'importance du recul (l'introspection) qui permet à la fois de mieux se comprendre et de mieux comprendre les autres (Channouf, 2004), mais ce recul ne peut que s'appuyer sur le discours et les comportements, c'est-à-dire sur ce qui est observable.

\subsection{Le discours : un reflet de ce qui est intériorisé}

Nous avons vu qu'un discours monolingue peut refléter des caractéristiques plurielles. La pensée n'est pas encodée. Quand elle le devient, elle suscite un idiolecte spécifique que l'on appelle discours intérieur (Vygotski, 1997). On 
pourrait, de ce fait, avancer que la production translangagière reflète la pluralité et la créativité de l'individu : elle lui permet d'aller au-delà de l'unicité des codes, des normes et des références. Si Wei (2017) nous dit que les pluriculturels/lingues construisent constamment leurs identités socioculturelles et leurs valeurs au travers d'une production translangagière, nous dirions plutôt que cette production reflète et traduit leur ressenti et les comportements qui en découlent et la façon dont le ressenti et les comportements sont interprétés dépendent des positions épistémologiques de ceux qui les interprètent, et donc de leur conception de ce que « culture » recouvre.

\subsection{Remise en cause du différentialisme}

Bayart (2002) affirme que le culturalisme (le recours à la culture dans son versant figé pour expliquer l'autre) a trois points contestables : (1) croire qu'une culture est un ensemble de représentations stables ; (2) considérer qu'elle est fermée sur elle-même ; (3) postuler qu'elle détermine une orientation d'actions précise. Ces trois points sont en contradiction avec ce qui précède, en particulier avec ce que postule l'énaction, puisque tout individu a sa spécificité même dans un groupe apparemment monoculturel (voir White, 2018). Dervin (2011) rappelle que les discours des couples qu'il observe ne se réfèrent pas constamment à la culture. S'ils le font, les participants affirment que c'est une forme de jeu pour faciliter la co-construction de la culture de l'échange et la mise en scène de leurs identités : il s'agit de jouer à être interculturel, un jeu que décrit Axelson (2007).

Le différentialisme à outrance, en particulier la culture comme explication principale des problèmes dans les couples, est ainsi contesté par les interviewés pour qui leurs comportements sont plus dû au fait qu'ils sont des êtres subjectifs, humains et sociaux qu'à leur origine culturelle (Dervin, 2011),

Dans les approches dites culturalistes de l'interculturel, l'individu disparait au profit d'éléments culturel « extérieurs » qui gouverneraient ses pensées, ses actes, ses opinions (White, 2011). L'identité est vue comme solide (Bauman, 2004 ; Chauvier, 2011 ; White, 2018). Une tendance contraire se développe depuis des années : le contexte est pris en compte, au-delà de la culture, à travers une approche " molle ", où l'individu est au centre des analyses (Dervin, 2011). Par contre, le caractère intersubjectif des rencontres et l'inévitable co-construction de ce qui est dit, fait, mis en scène, le fait que le discours structure l'échange et les identités, est laissé de côté.

Ces deux visions réifiées, généralisantes sont contestées pour être remplacées par une démarche herméneutique, intersubjective et contextualisée (Briedenbach \& Nyíri, 2009). Bensa (2010 : 79) affirme : « il n’y a pas de différence culturelle, il y a des différences d'histoire qui portent sur le type de ressources 
que les acteurs mobilisent ici et là-bas ". Le caractère intersubjectif des rencontres déclenche, en cohérence avec l'énaction, un processus de transformation qui ne correspond pas à une culture unique et figée. Néanmoins, il parait difficile de ne pas trouver des traces des divers environnements fréquentés chez les individus, traces qui expliquent que l'on généralise parfois à outrance ce que l'on appelle les traits nationaux.

\subsection{Traiter le relationnel sans « culture » ne répond pas à toutes les questions}

Dans les interviews de Dervin (2011), les participants, rejoignant certains auteurs, semblent penser que l'interculturel en tant que résultat de rapports coconstructifs devrait être traité " sans culture » (Dervin, 2011).

On peut imaginer que, selon Dervin (2011), sur le plan culturel :

- il y a métissage plus qu'essentialisme ;

- l'identité se construit au cours des interactions. Dervin (2011) parle d'identification comme processus ;

- il y aurait culturalité et non plus culture ;

- le construit de culture figée s'oppose à des processus soumis aux effets des interactions.

Les études et les données de Baena (2006) et Dervin (2011) confirment la suggestion méthodologique de Bensa de " s'affranchir de l'idée absurde d'une adhésion pleine et entière des acteurs à leur propre monde, sans que jamais leur perplexité, leur questionnement, leur éloignement relatif par rapport à ce qu'ils vivent, ne soient examinés " (Bensa, 2010, p. 36 -37). On comprend que le discours traduit cette complexité dynamique qui s'inscrit dans le vécu des individus et que nous appelons comportements transculturels.

Néanmoins, dans les interviews de Dervin (2011), certaines caractéristiques émergent dont il serait intéressant de déterminer l'origine et ce qui les cause :

- le rapport à la norme;

- le recours à des adjectifs évaluatifs tels que naturel, confortable, incorrect;

- le perfectionnisme;

- les contradictions internes;

- la langue des émotions;

- la dépendance et l'indépendance culturelles.

Cette origine peut être multiple pour chacun de ces items, et certainement différente pour chaque élément de la liste, mais elle est liée au vécu de l'individu, à son ressenti, et à la façon dont son ressenti l'a amené à modifier ses comportements et son discours, et sans doute aussi aux environnements qu'il a connus (ce que certains appellent les cultures qu'il a fréquentées). Tout 
en acceptant que le différentialisme et le culturalisme ont des limites évidentes, il parait utile de comprendre comment les comportements et les discours de chacun reflètent certaines caractéristiques des différents milieux qu'il a fréquentés, cela favorisera l'introspection, mais aussi la médiation, à condition bien sûr de ne pas se laisser aller à des interprétations hâtives...

\section{Positionnement}

Nous avons vu que, contrairement aux représentations traditionnelles, la pensée véritable procède par des va-et-vient et par sérendipité. La dynamique de la sérendipité, plus récurrente dans les sciences dites dures et les techniques, se retrouve également dans le domaine de la recherche en sciences sociales. En sociologie, des chercheurs de l'école de Chicago (voir Shepherd \& Rothenbuhler 2001) l'ont présentée comme une méthode scientifique propre à la théorie ancrée (grounded) et en ont proposé un usage plus systématique. Les débats actuels sur les moteurs de recherche (Johnson 2010) comme outils de sérendipité soulignent l'importance de la réflexion et de la validation scientifique dans la confirmation de toute découverte.

II nous semble que l'apport principal de notre parcours transdisciplinaire relève de la sérendipité. En rencontrant un construit rarement mentionné dans notre domaine, la théorie de l'esprit (compréhension sociale), nous nous sommes interrogés sur la pertinence de le distinguer de la compétence interculturelle. Selon Zarate (2002), la compétence interculturelle se construit dans la mise en relation de langues et de cultures, auprès d'acteurs sociaux qui les perçoivent comme distinctes, et non pas uniquement sur la base des différences. Son but principal est de prévenir, d'identifier et de réguler les malentendus et les difficultés de la communication dus à des décalages de schèmes interprétatifs, voire à des préjugés (stéréotypes, etc.). Elle est donc ce qui permet de comprendre et de gérer des rencontres avec des individus issus d'environnements très différents. On peut postuler qu'elle relève des même processus et stratégies que ce qui est plus généralement appelé théorie de l'esprit.

II nous semble que les préoccupations immédiates de notre domaine et un certain manque d'intérêt pour la psychologie ont fait négliger que toutes les interactions humaines relèvent des mêmes processus et que, si les référents dits " culturels » sont différents, cela ne signifie pas que les processus le sont. De plus, chaque individu construit langue(s) et culture(s) à sa façon, ce qui complexifie la mise en relation de langues et de cultures qu'avance Zarate (2002). L'idiographie des constructions individuelles confirme ainsi nos positions épistémologiques sur le caractère nécessairement situé de la linguistique appliquée (Condamines et Narcy-Combes, 2015). Comment en effet établir des lois universelles au niveau des relations humaines? 


\section{Conclusion}

Cette réflexion théorique nous rappelle que chacun :

- construit son monde en fonction de ce que déclenchent les rencontres et les environnements de sa vie ;

- réagit à lui-même et aux autres et agit avec eux en fonction de la théorie de l'esprit (compréhension sociale) qu'il s'est construite ;

- se comprend mieux et comprend en quoi il a bien ou mal interprété les autres et ce qu'il se passe autour de lui et en quoi ses (ré)actions étaient à propos ou non dans la situation vécue grâce à l'introspection.

En apparence cette réflexion est loin des préoccupations des enseignants de langues, mais dans les faits, ce n'est pas sûr. En effet, elle permet :

- de mieux comprendre ce qui anime les apprenants et les enseignants et de ne pas mettre les difficultés des rencontres pluriculturelles sur un plan différent de celles que révèlent les rencontres habituelles ;

- d'étudier les contextes dans une approche ascendante et participative sans succomber à un différentialisme rigide ;

- de relativiser le contexte (pluri)culturel en prenant en compte son dynamisme ;

- de mettre en place des médiations plus sensibles aux identités et aux comportements pluriels.

Et plus fondamentalement, elle souligne combien la construction des individus est complexe et dynamique et qu'il importe néanmoins de la comprendre et de la respecter afin que les jeux émotionnels ne contrecarrent pas le développement langagier et pluriculturel de chacun. Au niveau de la recherche et de la pédagogie, il semble que notre domaine gagnerait à collaborer avec des psychologues sociaux et qu'une sensibilisation à la compréhension sociale dès les premières années de scolarité faciliterait les rencontres interculturelles qui ne seraient plus aussi déstabilisantes dans la mesure où chacun serait prévenu de ce qui se passe en lui.

II reste à valider ces hypothèses et à déterminer comment, dans les dispositifs scolaires, généraliser cette sensibilisation qui, pour le moment, relève encore souvent de la psychologie clinique. Des recherches ouvrent des pistes, comme celle de White (2018), et d'Ennassiri (2014) qui a mis en avant, pour des étudiants en mobilité, le rôle de ce qu'il appelle métacognition et que nous appellerions introspection. Il conviendrait de commencer ce travail plus tôt dans la scolarisation des individus. Au vu de ce qui a été abordé dans l'introduction, il serait peut-être possible ainsi de prévenir plutôt que guérir les inconforts que peuvent susciter les rencontres humaines. 


\section{BIBLIOGRAPHIE}

Axelson E. (2007), Vocatives: a double-edged strategy in intercultural discourse among graduate students (dans) «Pragmatics ", n 17(1), p. 95-122.

Baena R. (dir.) (2006), Transculturing Auto/biography: Forms of Life Writing. New York : Routledge.

Bakhtine M. (1977), Marxisme et philosophie du langage. Paris : Minuit.

Bauman Z. (2004), Identity. Cambridge : Polity.

Bayart J.-F. (2002), The illusion of cultural identity. Chicago : University of Chicago Press.

Bensa A. (2010), Après Lévi-Strauss, pour une anthropologie à taille humaine. Paris : Textuel.

Bender A., Beller S. (2013), Cognition is ... Fundamentally Cultural (dans) « Behavioral sciences (Basel, Switzerland) ", $n^{\circ} 3(1)$, p. 42-54.

Breidenbach J., Nyíri P. (2009), Seeing culture everywhere. Seattle : Washington University Press.

Carstensen L. L. (1995), Evidence for a life-span theory of socioemotional selectivity (dans) "Psychological Science », $\mathrm{n}^{\circ}$ 4, p. 151-156.

Chang W. W. (2017), Approaches for Developing Intercultural Competence: An

Extended Learning Model With Implications From Cultural Neuroscience

(dans) " Human Resource Development Review », n 16(2), p. 158-175.

Channouf A. (2004), Les influences inconscientes. Paris: Armand Colin.

Chauvier E. (2011), Anthropologie de l'ordinaire. Toulouse : Anacharsis.

Cicurel F., Narcy-Combes J.-P. (2014), Quelle complémentarité entre les savoirs $d^{\prime}$ action et les savoirs théoriques ? Quelques significations à attribuer à I'action enseignante (dans) Aguilar J., Brudermann C., Leclère M. (dir.), Langues, cultures et pratiques en contexte : interrogations didactiques.

Paris : Riveneuve, p. 347-367.

Clot Y. (2008), Travail et pouvoir d'agir. Paris : PUF.

Condamines A., Narcy-Combes J.-P. (2015), La linguistique appliquée comme science située (dans) Carton F., Narcy-Combes J.-P., Narcy-Combes M.F., Toffoli D. (dir.), Cultures de recherche en linguistique appliquée. Paris : Riveneuve éditions, p. 209-229.

Damasio A. (2010), L'autre moi-même. La construction du cerveau conscient.

Paris : Odile Jacob.

Dehaene S. (2007), Les neurones de la lecture. Paris : Odile Jacob.

Dervin F. (2011), Les identités des couples interculturels. En finir vraiment avec la culture ? Paris : L'Harmattan.

Descola P. (2005), Par-delà nature et culture. Paris : Gallimard. 
Duclos A. M. (2015), Resistance to change: an outdated and invalid concept in education. (dans) "Psychologie \& Éducation ", n² 2015-1, p. 33-45.

Eagleman D. (2012), Incognito. Les vies secrètes du cerveau. Paris : Robert Laffont. Ennassiri H. (2014), Pour une conceptualisation de la communication interculturelle : choix théoriques, enjeux et perspectives. Thèse de l'université d'El Jadida.

Freze M., Zapf D. (1994), Action as the core of work psychology: a German approach (dans) « Handbook of Industrial and Organizational Psychology ", $n^{\circ} 4$, p. 271-340.

García O. (2009), Bilingual Education in the 21st Century: A Global Perspective. Malden, MA : Wiley/Blackwell.

Goffman E. (1974), Les rites d'interaction. Paris : les Éditions de Minuit. Grosjean F. (2008), Studying Bilinguals. Oxford : Oxford University Press. Guillaume C., Eustachen F., Desgrandes B. (2009), L'effet de positivité : un aspect intrigant du vieillissement (dans) "Revue de Neuropsychologie ", $\mathrm{n}^{\circ} 1$ (3), p. 247-253.

Gumperz J., Levinson, S. (dir.) (1996), Rethinking Linguistic Relativity. Cambridge : Cambridge University Press.

Habermas L. (1984), Discours, travail et polyfocalisation de l'action (dans) Habermas L. (dir.), The Theory of Communicative Action, volume I. Boston, $M A$ : Beacon Press.

Halliday M. A. K. (1975), Learning how to mean : Explorations in the development of language. London: Edward Arnold.

Jardou A. (2018), Compétence de communication interculturelle et mobilité étudiante : le cas des apprenants primoarrivants en France et des classes plurilingues et multiculturelles de FLE. Thèse de l'université Grenoble-Alpes.

Kaptelinin V. (1996), Activity Theory: Implications for Human Computer Interaction (dans) Nardi B. (dir.), Context and Consciousness: Activity Theory and Human-Computer Interaction. Cambridge, MA : MIT Press, p. 103-116.

Knight C., Power C. (2011), Social conditions for the evolutionary emergence of language (dans) Tallerman M., Gibson K. (dir.), Handbook of Language Evolution. Oxford : Oxford University Press, p. 346-349.

Lahire B. (2001), L'homme pluriel, les ressorts de l'action. Paris : Nathan. LeDoux J. (2003), Neurobiologie de la personnalité. Paris : Odile Jacob.

Lenoir Y. (2007), L'habitus dans l'œuvre de Pierre Bourdieu : un concept central dans sa théorie de la pratique à prendre en compte pour analyser les pratiques d'enseignement. Document du CRI et de la CRCIE, $\mathrm{n}^{\circ} 1$. Université de Sherbrooke.

Leontiev A. N. (2009), Activity Consciousness and Personality. Pacifica : Marxists Internet Archive. 
Moro M.R. (2010), Grandir en situation transculturelle. Des enfants qui appartiennent à plusieurs mondes. Paris : Fabert / Yakape.be.

Narcy-Combes J.-P. (2005). Didactique des langues et TIC, vers une rechercheaction responsable. Paris : Ophrys.

Narcy-Combes J.-P. (2018), Transculturing : un construit pour découvrir les ressorts du translanguaging (dans) « Language Education and Multilingualism ", n¹/2018, p. 52-65.

Narcy-Combes J.-P., Narcy-Combes M.-F. (2019), Cognition et personnalité dans I'apprentissage des langues. Relier théorie et pratique. Paris : Didier.

Narcy-Combes, M.-F. (2018), La transdisciplinarité dans l'intervention en linguistique appliquée (dans) «Étude de Linguistique Appliquée », n90, p. 183-195.

Norman, D. A. (1993), Things That Make Us Smart: Defending Human Attributes in the Age of the Machine. New York : Addison-Wesley Publishing Company.

Okon-Singer H., Hendler T., Pessoa L., Shackman A. J. (2015), The neurobiology of emotion - cognition interactions: Fundamental questions and strategies for future research (dans) "Frontiers in Human Neuroscience ", $n^{\circ} 9$, article 58. Payen V. (2011), Motivation d'approche et d'évitement : effets psychophysiologiques de la couleur rouge sur les processus cognitifs et moteurs. Thèse de l'Université de Toulon.

Perrenoud Ph. (1996), Enseigner. Agir dans I'urgence, décider dans l'incertitude. Paris : ESF.

Puren C. (2002), Perspectives actionnelles et perspectives culturelles en didactique des langues-cultures : vers une perspective co-actionnelle co-culturelle (dans) "Les Langues modernes ", $n^{\circ} 3$, p. 55-71.

Rabardel P. (2005), Instrument, activité et développement du pouvoir d'agir (dans) Rabardel P., Pastré P. (dir), Modèles du sujet pour la conception. Dialectiques activités développement. Toulouse : Octarès.

Rousset S. (2000), Les conceptions "système unique" de la mémoire : aspects théoriques (dans) "Revue de neuropsychologie ", n 10(1), p. 27-51.

Saïd E.W. (2002), À Contre-voie. Mémoires, [Out of place. A Memoir, 1999] traduction de Brigitte Caland et Isabelle Genet. Paris : Le Serpent à Plumes.

Sapir E. (1985), Selected Writings of Edward Sapir in Language, Culture and Personality. California : University of California Press.

Scheler M. (2003), Nature et formes de la sympathie. Contribution à l'étude des lois de la vie affective, trad. M. Lefebvre. Paris : Payot \& Rivages.

Schultz W., Tremblay L., Hollerman J. R. (2000), Reward Processing in Primate Orbitofrontal Cortex and Basal Ganglia (dans) "Cerebral Cortex ", $\mathrm{n}^{\circ} 10$ (3), p. 272-283.

Shahaeian A., Nielsen M., Peterson C. C. (2013), Cultural and Family Influences on Children's Theory of Mind Development: A Comparison of Australian 
and Iranian School-Age Children (dans) « Journal of Cross-Cultural Psychology ", vol.45, $n^{\circ} 4$, p. 555-568.

Varela F. (1993), L'Inscription corporelle de l'esprit. Paris : Seuil.

Vygotski L. (1997/1934), Pensée et langage, 3ème édition. Paris : La Dispute.

Wehbe O. (2017), Questions que pose une didactique plurilingue au Liban, pratiques et représentations. Thèse de l'université Sorbonne Nouvelle - Paris 3.

Wei L. (2017), Translanguaging as a Practical Theory of Language (dans) « Applied Linguistics $"$, vol. 39, $\mathrm{n}^{\circ} 1$, p. 9-30.

White J. (2018), Apprendre à rencontrer l'autre - les effets de la réflexion guidée sur le discours des étudiants universitaires en mobilité. Thèse de I'université Paul Valéry. Montpellier III.

Wittgenstein L. (1980), Grammaire philosophique. Paris : Gallimard.

Xue L. (2016), Aspects évolutifs de l'agir professoral dans le domaine de l'enseignement des langues. Une étude à travers les discours de verbalisation de six enseignants de français langue étrangère et de chinois langue étrangère. Thèse de l'Université Sorbonne Nouvelle-Paris 3.

Zarate G. (2002), Le compromis entre innovation européenne et tradition nationale dans l'actualisation d'un programme de langues vivantes (dans) Guidère M., \& Association des professeurs de langues vivantes de l'enseignement public (dir.), L'interculturel. Paris : Nathan, p. 5-14. 